\title{
Anomalous structure and dynamics of the Gaussian-core fluid
}

\author{
William P. Krekelberg, ${ }^{1, *}$ Tanuj Kumar, ${ }^{1}$ Jeetain Mittal, ${ }^{2, \dagger}$ Jeffrey R. Errington, ${ }^{3, \$}$ and Thomas M. Truskett ${ }^{1,4, \S}$ \\ ${ }^{1}$ Department of Chemical Engineering, University of Texas at Austin, Austin, Texas 78712, USA \\ ${ }^{2}$ Laboratory of Chemical Physics, NIDDK, National Institutes of Health, Bethesda, Maryland 20892-0520, USA \\ ${ }^{3}$ Department of Chemical and Biological Engineering, University at Buffalo, The State University of New York, \\ Buffalo, New York 14260-4200, USA \\ ${ }^{4}$ Institute for Theoretical Chemistry, University of Texas at Austin, Austin, Texas 78712, USA \\ (Received 29 December 2008; revised manuscript received 4 February 2009; published 18 March 2009)
}

\begin{abstract}
It is known that there are thermodynamic states for which the Gaussian-core fluid displays anomalous properties such as expansion upon isobaric cooling (density anomaly) and increased single-particle mobility upon isothermal compression (self-diffusivity anomaly). Here, we investigate how temperature and density affect its short-range translational structural order, as characterized by the two-body excess entropy. We find that there is a wide range of conditions for which the short-range translational order of the Gaussian-core fluid decreases upon isothermal compression (structural order anomaly). As we show, the origin of the structural anomaly is qualitatively similar to that of other anomalous fluids (e.g., water or colloids with short-range attractions) and is connected to how compression affects static correlations at different length scales. Interestingly, we find that the self-diffusivity of the Gaussian-core fluid obeys a scaling relationship with the two-body excess entropy that is very similar to the one observed for a variety of simple liquids. One consequence of this relationship is that the state points for which structural, self-diffusivity, and density anomalies of the Gaussiancore fluid occur appear as cascading regions on the temperature-density plane; a phenomenon observed earlier for models of waterlike fluids. There are, however, key differences between the anomalies of Gaussian-core and waterlike fluids, and we discuss how those can be qualitatively understood by considering the respective interparticle potentials of these models. Finally, we note that the self-diffusivity of the Gaussian-core fluid obeys different scaling laws depending on whether the two-body or total excess entropy is considered. This finding, which deserves more comprehensive future study, appears to underscore the significance of higherbody correlations for the behavior of fluids with bounded interactions.
\end{abstract}

DOI: 10.1103/PhysRevE.79.031203

PACS number(s): 61.20.Ja, 83.10.Rs, 65.40.gd, 66.10.C-

Model pair potentials that describe the interactions between particles of simple atomic and molecular liquids generally have steeply repulsive short-range components that diverge as two particles approach one another, qualitatively capturing the increase in energy that accompanies overlap of their electronic clouds. However, the effective pair interactions between the centers of mass of two polymer chains in solution (or molecular aggregates such as micelles) are often more accurately represented by softer bounded potentials [1], since they describe "particles" that are inherently interpenetrable. A simple example of a bounded interaction is given by the Gaussian-core (GC) model [2]. It is defined by a pair potential of the following form $\phi(r)=\epsilon \exp \left[-(r / \sigma)^{2}\right]$, where $r$ is the distance between particle centers, and $\epsilon$ and $\sigma$ characterize the amplitude and width of the interaction profile, respectively. Understanding the differences between the collective properties of penetrable particles with bounded effective potentials and those with steeply repulsive interactions is a basic problem in the study of soft condensed matter (see, e.g., [3-5]).

The behavior of the GC fluid is indeed unusual when compared to most atomic or molecular fluids. For example,

\footnotetext{
*wpkrekelberg@gmail.com

†jeetain@ @elix.nih.gov

\#jerring@buffalo.edu

${ }^{\S}$ Corresponding author; truskett@che.utexas.edu
}

there are state points for which its thermal-expansion coefficient is negative (density anomaly) [6] and for which its single-particle dynamics increase upon isothermal compression (diffusivity anomaly) [7]. At sufficiently low temperature, the fluid also exhibits re-entrant melting behavior $[2,3,8]$. Anomalous changes of self-diffusivity with density or temperature are known to occur in other model systems that qualitatively mimic either the behaviors of (i) liquid water or (ii) suspensions of colloids with short-range interparticle attractions, respectively. A series of theoretical studies has shown that those changes for both systems of type (i) [9-24] and type (ii) $[16,23,25]$ are strongly correlated with anomalous trends in the short-range translational structural order of the respective fluids. Moreover, an analysis of recent Brownian dynamics simulations of the GC fluid [26] suggested a qualitative (but not quantitative) connection between the anomalous density dependencies of the selfdiffusivity and the pair-correlation function. Here, we carry out an extensive molecular simulation study of the GC fluid to investigate whether a quantitative link between its dynamics and its short-range translational order can be established.

To compute the properties of the GC model, we perform molecular-dynamics simulations in the microcanonical ensemble using $N=1000$ particles in a cubic cell with periodic boundary conditions. For simplicity of notation, we implicitly nondimensionalize all quantities by appropriate combinations of the characteristic length scale $l_{c}=\sigma$ and time scale $\tau_{c}=\sqrt{m \sigma^{2} / \epsilon}$, where $m$ is the mass of a particle. We integrate 

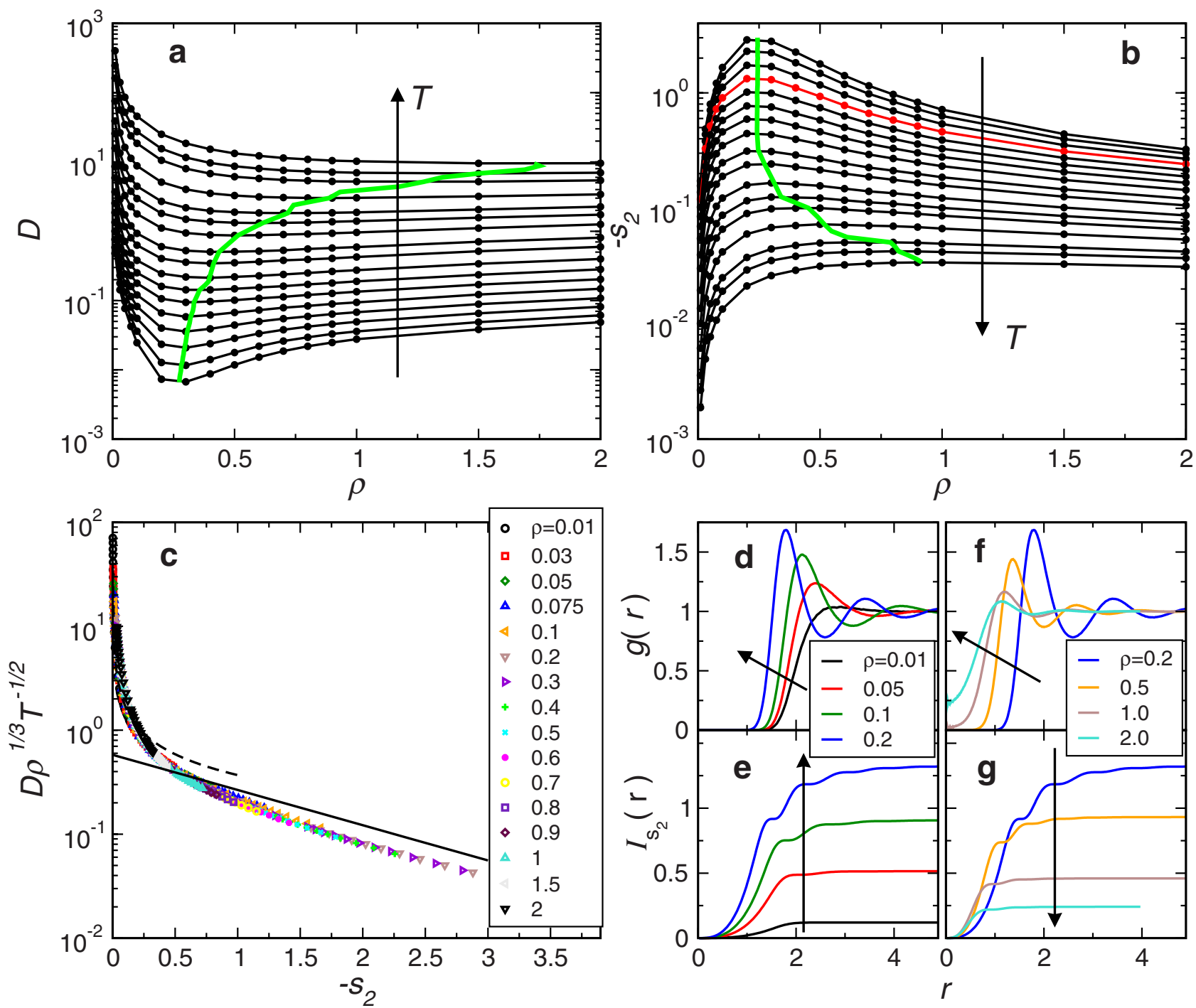

FIG. 1. (Color online) (a) Self-diffusivity $D$ and (b) structural order metric $-s_{2}$ versus density $\rho$ at temperatures $T=0.01,0.013,0.018$, $0.025,0.035,0.05,0.07,0.1,0.15,0.2,0.3,0.4,0.5,0.7,1.0,1.2$, and 1.5 for the GC fluid. Arrows indicate increasing temperature. Solid green (gray) lines in (a) and (b) correspond to minima in $D$ and maximum in $-s_{2}$, respectively. (c) Rosenfeld scaled diffusivity versus $-s_{2}$ at all densities and temperatures studied. The solid line represents the empirical Rosenfeld scaling law $0.58 \exp \left(0.78 s_{2}\right)$ and the dashed curve represents the low-density behavior expected for soft spheres $0.37\left(-s_{2}\right)^{-2 / 3}$. (Lower right panel) Pair correlation function $g(r)$ and cumulative order integral $I_{s_{2}}(r)$ along the isotherm $T=0.025$ [red curve in (b)] for two density ranges [(d) and (e)] $\rho \leq 0.2$ [below the maximum in $\left.-s_{2}(\rho)\right]$ and $[(\mathrm{f})$ and $(\mathrm{g})] \rho \geq 0.2$ [above the maximum in $\left.-s_{2}(\rho)\right]$. In $(\mathrm{d})-(\mathrm{g})$, arrows indicate increasing $\rho$.

the equations of motion using the velocity-Verlet method [27] with time step $\delta t=0.05$, and we cut the GC potential at $r_{\text {cut }}=3.2$. We calculate self-diffusivity by fitting the long-time $(t \gg 1)$ behavior of the mean-squared displacement $\left\langle\delta \mathbf{r}^{2}(t)\right\rangle$ to the Einstein formula $6 D t=\left\langle\delta \mathbf{r}^{2}(t)\right\rangle$. We investigate temperatures between $T=0.01$ and 1.5 and number densities between $\rho=0.01$ and 2.0 [28], parameters that span the part of phase space where the aforementioned anomalous behavior are known to occur for the GC fluid [7].

Figure 1(a) displays the self-diffusivity $D$ of the GC fluid versus density $\rho$ for several isotherms. Like in simpler fluids, compressing the low-density GC fluid initially decreases $D$. However, as noted previously [7], compression can eventually lead to an anomalous increase in $D$ at sufficiently high particle density. In fact, for a given $T$, one can view the density at which the minimum in $D$ occurs as a boundary between regions of "normal" and anomalous dynamic behavior. This boundary is approximately indicated by the green (gray) line in Fig. 1(a) [29].

To address the question of whether this anomalous dynamic behavior is related to the structural order, we first investigate the behavior of the two-body contribution to the excess entropy (relative to an ideal gas at the same $T$ and $\rho$ ) defined as $[30,31]$

$$
s_{2} \equiv-2 \pi \rho \int_{0}^{\infty} r^{2}\{g(r) \ln g(r)-[g(r)-1]\} d r,
$$

where $g(r)$ is the pair-correlation function (PCF). The quantity $-s_{2}$ effectively characterizes the degree of pair translational order present in the fluid [32]. Figure 1(b) displays $-s_{2}$ versus $\rho$ for several isotherms. Like in simple fluids, com- 
pressing the low-density GC fluid initially increases the structural order. However, further increasing the density eventually leads to a qualitative change in behavior. Specifically, the GC fluid begins to lose structural order upon compression at sufficiently high density; a trend that does not generally occur in atomic fluids $[31,32]$. Thus, for a particular $T$, the density corresponding to the maximum in $-s_{2}$ can similarly be viewed as a boundary between state points of "normal" and anomalous structural order. This boundary is approximately indicated by the green (gray) line in Fig. 1(b) [29].

Together, Figs. 1(a) and 1(b) suggest a strong correlation between dynamics and structural order for the GC fluid. Given the recent results from computer simulations $[16,18,24,25,33-42]$ and experiments $[43,44]$ which show a clear connection between dynamics and excess entropy for a wide variety of systems, this correlation may not be particularly surprising. In fact, an approximate scaling law relating transport coefficients and excess entropy per particle $s^{\text {ex }}$ was first noted by Rosenfeld [33,35]. Specifically, Rosenfeld observed that the following scaled form of self-diffusivity $D_{R}$ $=D \rho^{1 / 3} T^{-1 / 2}$ is a quasiuniversal function of $s^{\mathrm{ex}}$ for a variety of models of simple atomic fluids. In other words, the dependencies of $D$ on $T$ and $\rho$ can essentially be reduced to how these variables affect $s^{\text {ex }}$. For simple liquid systems at low to intermediate densities, most of the excess entropy $\left(s^{\mathrm{ex}}\right)$ comes from the two-body contribution $\left(s_{2}\right)$ [31], and thus the quasiuniversal excess entropy scaling indicates that their transport coefficients are closely linked to $g(r)$ (see also, e.g., [45]).

For atomic fluids, the scaling law relating $D$ and $s^{\text {ex }}$ closely tracks different functional forms as $-s^{\mathrm{ex}}$ goes from lower to higher values. For very low $-s^{\mathrm{ex}}$ (i.e., a dilute gas), the following power-law form follows from Enskog theory for $D$ and a second-virial approximation for $s^{\text {ex }}$ (see [35]),

$$
D \rho^{1 / 3} T^{-1 / 2} \approx \alpha\left(-s^{\mathrm{ex}}\right)^{\beta}
$$

where $\alpha$ and $\beta$ are constants. At higher $-s^{\text {ex }}$ (e.g., a fluid near the freezing transition), the following exponential relation has been empirically observed [35]

$$
D \rho^{1 / 3} T^{-1 / 2} \approx A \exp \left[B s^{\mathrm{ex}}\right]
$$

where $A$ and $B$ are constants.

Figure 1(c) displays the scaled self-diffusion coefficient $D_{R}$ versus the two-body estimate of the excess entropy $-s_{2}$. Note that the dependencies of the Rosenfeld self-diffusivity $D_{R}$ on $T$ and $\rho$ for the GC fluid also collapse onto a single master curve when plotted versus $-s_{2}$. For comparison, Eq. (2) with parameters that fit the simulation data for a variety of soft-sphere model systems $(\alpha=0.37, \beta=-2 / 3$ [35] and $A=0.58, B=0.78$ [33]) is also shown in Fig. 1(c). The main point to note here is that despite small quantitative differences, the form of the relationship between the diffusivity and excess entropy is virtually the same, whether one considers the anomalous GC fluid or a model for a simple atomic fluid. In other words, despite the anomalous dependency of $D$ upon $\rho$ for the GC fluid, the relationship between $D$ and $s_{2}$ is perfectly normal.
Given this basic link between anomalous dynamics and structure, we now investigate the molecular origins of the trends in $-s_{2}$. To this end, we study the PCF and the related cumulative order integral $I_{s_{2}}(r)$ defined as [25]

$$
I_{s_{2}}(r) \equiv 2 \pi \rho \int_{0}^{r} r^{\prime 2}\left\{g\left(r^{\prime}\right) \ln g\left(r^{\prime}\right)-\left[g\left(r^{\prime}\right)-1\right]\right\} d r^{\prime} .
$$

Note that one recovers $-s_{2}$ from this integral in the large $r$ limit. The quantity $I_{s_{2}}(r)$ characterizes the average amount of translational ordering on length scales smaller than $r$ surrounding a particle. Figures $1(\mathrm{~d})-1(\mathrm{~g})$ show the behavior of $g(r)$ and $I_{s_{2}}(r)$ for selected state points along the lowtemperature $T=0.025$ isotherm [red curve in Fig. 1(b)]. They divide the behavior into two qualitatively different regions: (1) the normal increase of $-s_{2}$ with $\rho$ at low densities $[\rho$ $\leq 0.2$, Figs. 1(d) and 1(e)] and (2) the anomalous decrease of $-s_{2}$ with $\rho$ at high densities $[\rho \geq 0.2$, Figs. $1(\mathrm{f})$ and $1(\mathrm{~g})]$.

Inspection of Fig. 1(d) shows that compression of the low-density GC fluid leads to an increased degree of translational order reflected by the formation of distinct first, second, and more distant coordination shells in $g(r)$. This increase in "packing" order around the particles [see Fig. 1(e)] is also observed in hard-sphere fluids and simple atomic systems, and it is a reflection of the fact that local interparticle correlations necessarily build up as density increases in order for particles to avoid overlap with one another. At sufficiently low pressures (low densities) and low temperature, the effective repulsive "core" of the GC potential acts like a hard-sphere interaction and that is the primary reason that the structural trends of the GC fluid under these conditions mimic those of simple atomic systems.

Further compression of the GC fluid [region (2), $\rho \geq 0.2$ ] leads to an anomalous decrease in structural order [Fig. 1(b)]. In this higher pressure, higher density region of phase space, the repulsion of the GC interparticle potential is too soft to effectively prevent interparticle overlap. In fact, because the system only pays a finite potential-energy penalty for each overlap, and since avoiding overlaps carries a substantial entropic penalty, the system evolves toward a more uniform average structure at sufficiently high density. In the limit of infinitely high density, the particle centers of the GC fluid will adopt a Poisson distribution (i.e., a high-density ideal gas) [3]. As can be seen in Fig. 1(f), these compressioninduced changes manifest as a shifting in, flattening, and broadening of the coordination shells in $g(r)$. Figure $1(\mathrm{~g})$ shows that while finite penetrability gives rise to a small increase in order at small $r$ (inside the core), the overall behavior is dominated by the corresponding disordering that occurs at larger $r$.

The microscopic origins of the structural behavior of the GC fluid share a qualitative similarity with those of the other anomalous fluids discussed earlier (liquid water and suspensions of colloids with short-range attractions) [23]. In the case of models that capture waterlike behavior, the compression of the low-temperature fluid can result in an anomalous translational disordering [9-11,16,18-20,22,37,42,46-49]. This decrease in structural order is primarily due to a shifting in, flattening, and broadening of the second coordination 


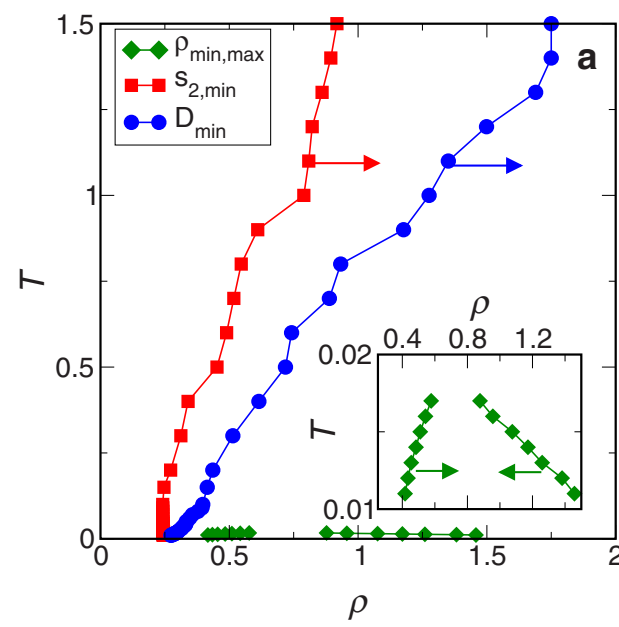

shell $[20,23]$, which is accompanied by the penetration of a fifth water molecule into the periphery of the first shell of otherwise tetrahedrally coordinated molecules (see, e.g., [20]). In the case of colloids with short-range attractions, increasing the strength of the attractive interactions (relative to $k_{B} T$ ) of the concentrated fluid can also give rise to an anomalous disordering $[16,25]$. This disordering is due to the fact that the short-range attractions favor near contact configurations of the particles. This disrupts the structure of the second and more distant coordination shells that otherwise naturally form when structure is determined primarily by the repulsive interactions (i.e., as in hard sphere or simple atomic fluids) [23]. Thus, in each of the above systems, there are two effects that are linked: (i) an increase in correlations at short length scales and (ii) an associated decrease in structural order at larger length scales. The latter effect dominates in these fluids, thus giving rise to their structurally anomalous behavior.

It is also interesting to ask about the relative placement of the boundaries for structural, diffusivity, and density anomalies on the temperature-density plane. Figure 2(a) shows those boundaries for the GC fluid as determined from our simulation data, and Fig. 2(b) provides an idealized schematic based on that data. The most striking feature is that the state points which exhibit the density anomaly (negative thermal-expansion coefficient) are a subset of those associated with the diffusivity anomaly, which, in turn, are a subset of those associated with the structural anomaly. This "cascade of anomalies" is qualitatively similar to that originally noticed for a molecular model of water by Errington and Debenedetti [9] and more recently observed in a wide class of model systems that exhibit waterlike behavior [10-15,17-22,47,48,50]. One qualitative difference between the GC fluid and water is that while water returns to normal structural and diffusivity trends at sufficiently high density, the GC fluid does not. This difference follows from the fact that water has steeply repulsive short-range interactions, which dominate its physics at sufficiently high densities (pressures). The repulsive core is, of course, absent in bounded potentials like the GC model, and thus those systems never "return" to hard-sphere-like structural and dynamic behavior at high density. However, like water, the GC fluid does return to normal (i.e., positive) thermal-expansion
FIG. 2. (Color online) (a) Boundaries of the structural, diffusivity, and density anomalies in the temperature-density plane, as calculated from simulation data. The inset shows the boundary of the region of density anomalies on an expanded scale. The symbols are the locations of the extrema and arrows are in the direction of the anomalous regions. (b) Schematic representation of anomalous regions.

$\rho$ behavior at sufficiently high density. This is consistent with the fact that the GC fluid's equation of state approaches a mean-field description in that limit [3]. Finally, we note that de Oliveira et al. [50] created a simple model for water with a spherically symmetric pair potential represented by a sum of a Lennard-Jones contribution (which contains the necessary repulsive core) and a Gaussian-core interaction. Given the discussion above, it should not be surprising that this generic type of hybrid model can qualitatively reproduce water's density, diffusivity, and structural anomalies [22,46-48].

It is also interesting to note that the state points where models of colloidal fluids with short-range attractions show that diffusivity anomalies are similarly a subset of the state points where they exhibit structural anomalies [25]. As has now been discussed extensively for models of water [17] and for models of colloids with short-range attractions [25], the "cascade of anomalies" has been shown to be qualitatively consistent with the behavior of the excess entropy, the empirical relationship between diffusivity and excess entropy, and the rigorous thermodynamic link between the excess entropy and the thermal-expansion coefficient.

Thus far, we have focused exclusively on the two-body contribution to the excess entropy $\left(s_{2}\right)$ of the GC fluid rather than the excess entropy itself $\left(s^{\mathrm{ex}}\right)$. The rationale for doing so is simple. The two-body quantity is a particularly convenient structural measure to compute and analyze given its straightforward connection to $g(r)$. Furthermore, as discussed above, $s_{2}$ is known to closely approximate $s^{\text {ex }}$ for simple fluids at low to intermediate density [31]. However, the GC fluid has structural behavior that is very different from that of simple atomic fluids. In fact, it is known that there are significant differences between $s_{2}$ and $s^{\text {ex }}$ for this system due to noncanceling effects from higher-order static correlations (3-body, 4-body, etc.) [51]. As an example, along the $T=0.025$ isotherm highlighted above, the ratio $s^{\mathrm{ex}} / s_{2}$ evaluates to approximately 1.7 at $\rho=0.3$ (where $-s^{\mathrm{ex}}$ reaches a maximum) and 3.0 at $\rho=1.0$. Thus, as a final point, we present in Fig. 3(a) the relationship between the Rosenfeld diffusivity $D_{R}$ $=D \rho^{1 / 3} T^{-1 / 2}$ and $-s^{\text {ex }}$ for all state points considered in this study of the GC fluid. We calculate $s^{\mathrm{ex}}$ in this study using grand-canonical transition-matrix Monte Carlo simulations [17,52-54]. 


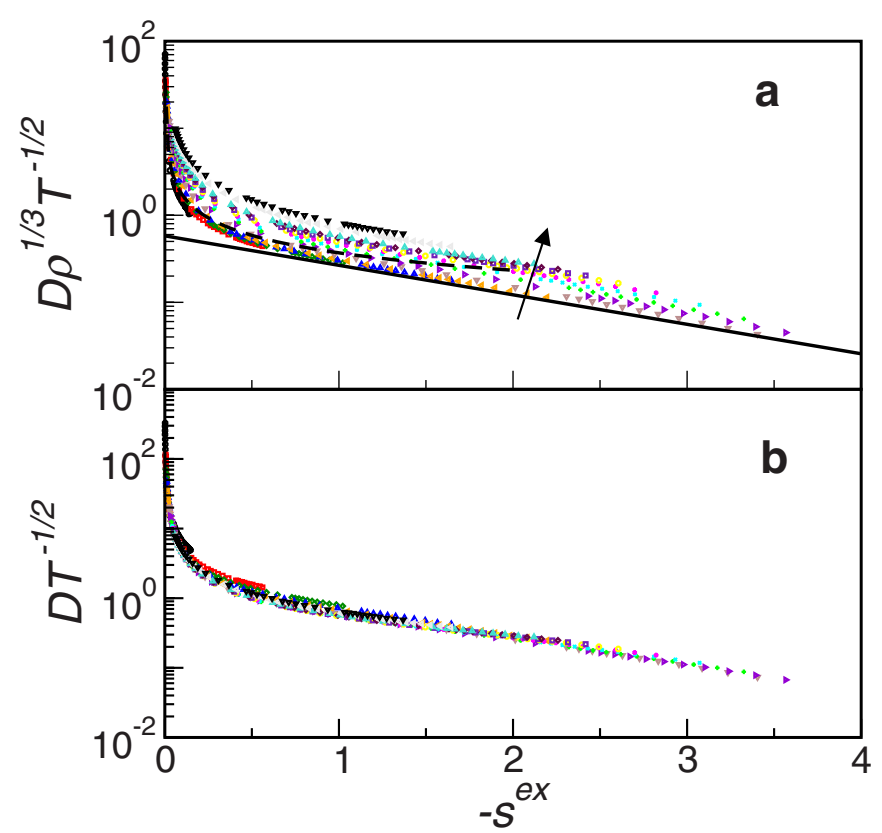

FIG. 3. (Color online) (a) Rosenfeld scaled diffusivity versus excess entropy $-s^{\mathrm{ex}}$. The solid and dashed curves, as well as the symbols, are the same as those in Fig. 1(c). The arrow is in the direction of increasing density. (b) Temperature scaled diffusivity $D T^{-1 / 2}$ versus $-s^{\text {ex }}$. The data represent all densities and temperatures considered in this study.

Note that for a given density, the relationship between $D_{R}$ and $-s^{\mathrm{ex}}$ is qualitatively similar to that of atomic fluids and hence also to the relationship between $D_{R}$ and $-s_{2}$ for the GC fluid shown in Fig. 1(c). However, the $D_{R}$ data at different densities do not collapse onto a single curve when plotted versus $-s^{\text {ex }}$ [contrast with Fig. 1(c)]. As expected, the lowdensity data are indeed accurately represented by Eq. (2) with parameters that describe soft-sphere model systems. However, as density is increased, $D_{R}$ becomes increasingly underestimated by the soft-sphere relation. Interestingly, as is shown in Fig. 3(b), the quantity $D T^{-1 / 2}$ does approximately collapse for all densities when plotted versus $-s^{\mathrm{ex}}$ for the GC fluid. At present, it is not known why this alternative scaling holds for this system or whether it can be expected to describe the behaviors of other fluids with bounded potentials (e.g., the penetrable sphere model [3]). We are currently car- rying out a systematic investigation of the thermodynamic and dynamic behavior of a variety of bounded potential fluids to explore this issue, and we will report our findings in a future study.

In conclusion, we present molecular simulation results which demonstrate that the GC fluid exhibits cascading regions of density, self-diffusivity, and structural (two-body excess entropy) anomalies in the temperature-density plane. Interestingly, an appropriately normalized self-diffusivity of the GC fluid follows a scaling with the two-body excess entropy that is similar to that observed for simple atomic fluids, which do not exhibit anomalies. The significance of this relation is that the self-diffusivity anomaly can be viewed as simply a reflection of the structural anomalies of the GC fluid. An analysis of the pair correlation function shows that the molecular origins of the structural anomalies of the GC fluid are easy to understand and are qualitatively similar to those of liquid water and colloids with short-range attractions. Specifically, changes in thermodynamic parameters give rise to an increase in pair correlations at short length scales and an associated disordering at larger length scales. For all of these fluids, the latter effect dominates, giving rise to the anomalous structural trends. Finally, we show that while the Rosenfeld diffusivity $\left(D_{R}=D \rho^{1 / 3} T^{-1 / 2}\right)$ is approximately a function of $s_{2}$ alone for the GC fluid, a different combination $\left(D T^{-1 / 2}\right)$ collapses as a function of $s^{\text {ex }}$. Whether this latter observation will generally hold for other fluids with bounded potentials is currently an open question which deserves more careful study.

We thank J. L. Carmer for useful discussions. We are also grateful to S. van Teeffelen, C. N. Likos, and H. Löwen for bringing Refs. $[4,26]$ to our attention. Two authors (T.M.T and J.R.E) acknowledge financial support of the National Science Foundation (Grant Nos. CTS-0448721 and CTS028772, respectively). One author (T.M.T.) also acknowledges support of the Welch Foundation (Grant No. F-1696) and the David and Lucile Packard Foundation. W.P.K. acknowledges the National Science Foundation GRF Program. J.M. acknowledges support by the National Institute of Diabetes and Digestive and Kidney Diseases Intramural Research Program. The Texas Advanced Computing Center (TACC) and University at Buffalo Center for Computational Research provided computational resources for this study.
[1] P. J. Flory and W. R. Krigbaum, J. Chem. Phys. 18, 1086 (1950).

[2] F. H. Stillinger, J. Chem. Phys. 65, 3968 (1976).

[3] A. Lang, C. N. Likos, M. Watzlawek, and H. Löwen, J. Phys.: Condens. Matter 12, 5087 (2000).

[4] C. N. Likos, B. M. Mladek, A. J. Moreno, D. Gottwald, and G. Kahl, Comput. Phys. Commun. 179, 71 (2008).

[5] C. E. Zachary, F. H. Stillinger, and S. Torquato, J. Chem. Phys. 128, 224505 (2008).

[6] F. H. Stillinger and D. K. Stillinger, Physica A 244, 358
(1997).

[7] P. Mausbach and H. O. May, Fluid Phase Equilib. 249, 17 (2006).

[8] S. Prestipino, F. Saija, and P. V. Giaquinta, Phys. Rev. E 71, 050102(R) (2005).

[9] J. R. Errington and P. G. Debenedetti, Nature (London) 409, 318 (2001).

[10] M. S. Shell, P. G. Debenedetti, and A. Z. Panagiotopoulos, Phys. Rev. E 66, 011202 (2002).

[11] T. M. Truskett and K. A. Dill, J. Phys. Chem. B 106, 11829 
(2002).

[12] P. Kumar, S. V. Buldyrev, F. Sciortino, E. Zaccarelli, and H. E. Stanley, Phys. Rev. E 72, 021501 (2005).

[13] R. Esposito, F. Saija, A. M. Saitta, and P. V. Giaquinta, Phys. Rev. E 73, 040502(R) (2006).

[14] P. A. Netz, S. V. Buldyrev, M. C. Barbosa, and H. E. Stanley, Phys. Rev. E 73, 061504 (2006).

[15] L. Xu, S. V. Buldyrev, C. A. Angell, and H. E. Stanley, Phys. Rev. E 74, 031108 (2006).

[16] J. Mittal, J. R. Errington, and T. M. Truskett, J. Phys. Chem. B 110, 18147 (2006).

[17] J. R. Errington, T. M. Truskett, and J. Mittal, J. Chem. Phys. 125, 244502 (2006).

[18] R. Sharma, S. N. Chakraborty, and C. Chakravarty, J. Chem. Phys. 125, 204501 (2006).

[19] A. Barros de Oliveira, M. C. Barbosa, and P. A. Netz, Physica A 386, 744 (2007).

[20] Z. Yan, S. V. Buldyrev, P. Kumar, N. Giovambattista, P. G. Debenedetti, and H. E. Stanley, Phys. Rev. E 76, 051201 (2007).

[21] M. M. Szortyka and M. C. Barbosa, Physica A 380, 27 (2007).

[22] A. Barros de Oliveira, G. Franzese, P. A. Netz, and M. C. Barbosa, J. Chem. Phys. 128, 064901 (2008).

[23] W. P. Krekelberg, J. Mittal, V. Ganesan, and T. M. Truskett, Phys. Rev. E 77, 041201 (2008).

[24] Z. Yan, S. V. Buldyrev, and H. E. Stanley, Phys. Rev. E 78, 051201 (2008).

[25] W. P. Krekelberg, J. Mittal, V. Ganesan, and T. M. Truskett, J. Chem. Phys. 127, 044502 (2007).

[26] H. Wensink, H. Löwen, M. Rex, C. Likos, and S. van Teeffelen, Comput. Phys. Commun. 179, 77 (2008).

[27] M. P. Allen and D. J. Tildesley, Computer Simulations of Liquids (Oxford University Press, New York, 1987).

[28] Specifically, we study reduced temperatures $T=0.01,0.011$, $0.012,0.013,0.014,0.015,0.016,0.017,0.018,0.019,0.02$, $0.025,0.03,0.035,0.04,0.045,0.05,0.06,0.07,0.08,0.09$, $0.1,0.15,0.2,0.3,0.4,0.5,0.6,0.7,0.8,0.9,1.0,1.1,1.2,1.3$, 1.4 , and 1.5 and reduced densities $\rho=0.01,0.03,0.05,0.075$, $0.1,0.2,0.3,0.4,0.5,0.6,0.7,0.8,0.9,1.0,1.5$, and 2.0.

[29] To calculate the location of the extrema, the raw simulation data along a given isotherm for densities just below at and just above the simulated extrema was fit to second degree polynomials. These polynomials were in turn used to calculate the locations of the extrema.

[30] R. E. Nettleton and M. S. Green, J. Chem. Phys. 29, 1365 (1958).

[31] A. Baranyai and D. J. Evans, Phys. Rev. A 40, 3817 (1989).

[32] T. M. Truskett, S. Torquato, and P. G. Debenedetti, Phys. Rev.
E 62, 993 (2000).

[33] Y. Rosenfeld, Phys. Rev. A 15, 2545 (1977).

[34] M. Dzugutov, Nature (London) 381, 137 (1996).

[35] Y. Rosenfeld, J. Phys.: Condens. Matter 11, 5415 (1999).

[36] J. Mittal, J. R. Errington, and T. M. Truskett, Phys. Rev. Lett. 96, 177804 (2006).

[37] J. Mittal, J. R. Errington, and T. M. Truskett, J. Chem. Phys. 125, 076102 (2006).

[38] J. Mittal, J. Errington, and T. Truskett, J. Phys. Chem. B 111 10054 (2007).

[39] J. Mittal, J. R. Errington, and T. M. Truskett, J. Chem. Phys. 126, 244708 (2007).

[40] J. Mittal, V. K. Shen, J. R. Errington, and T. M. Truskett, J. Chem. Phys. 127, 154513 (2007).

[41] G. Goel, W. P. Krekelberg, J. R. Errington, and T. M. Truskett, Phys. Rev. Lett. 100, 106001 (2008).

[42] M. Agarwal, R. Sharma, and C. Chakravarty, J. Chem. Phys. 127, 164502 (2007).

[43] E. H. Abramson and H. West-Foyle, Phys. Rev. E 77, 041202 (2008).

[44] E. H. Abramson, Phys. Rev. E 76, 051203 (2007).

[45] A. Samanta, Sk. Musharaf Ali, and S. K. Ghosh, Phys. Rev. Lett. 87, 245901 (2001).

[46] Z. Yan, S. V. Buldyrev, N. Giovambattista, and H. E. Stanley, Phys. Rev. Lett. 95, 130604 (2005).

[47] Z. Yan, S. V. Buldyrev, N. Giovambattista, P. G. Debenedetti, and H. E. Stanley, Phys. Rev. E 73, 051204 (2006).

[48] A. Barros de Oliveira, P. A. Netz, T. Colla, and M. C. Barbosa, J. Chem. Phys. 125, 124503 (2006).

[49] M. Agarwal and C. Chakravarty, J. Phys. Chem. B 111, 13294 (2007).

[50] A. Barros de Oliveira, P. A. Netz, T. Colla, and M. C. Barbosa, J. Chem. Phys. 124, 084505 (2006).

[51] P. V. Giaquinta and F. Saija, ChemPhysChem 6, 1768 (2005).

[52] J. R. Errington, J. Chem. Phys. 118, 9915 (2003).

[53] J. R. Errington, Phys. Rev. E 67, 012102 (2003).

[54] These simulations require fixed values of the activity $\xi$, volume $V$, and temperature $T$ as inputs. The activity is defined as $\xi=\Lambda^{-3} \exp \left(\mu / k_{B} T\right)$, where $\mu$ is the chemical potential and $\Lambda$ is the thermal de Broglie wavelength. For the GC-TMMC simulations employed here, we set $V=343$ and $\xi=1$, and we collect information over a range of densities spanning from $\rho=0.0$ to 2.0 at select values of the temperature. System size effects were examined by performing a subset of analogous calculations with $V=646$ and were found to be negligible. Thermodynamic properties at values of activity other than $\xi=1$ were readily obtained via the histogram reweighting technique. 